\title{
PIPSA، PROGRAMA INTEGRAL DE PROMOCIÓN DE SALUD DEL ADOLESCENTE
}

\section{Introducción}

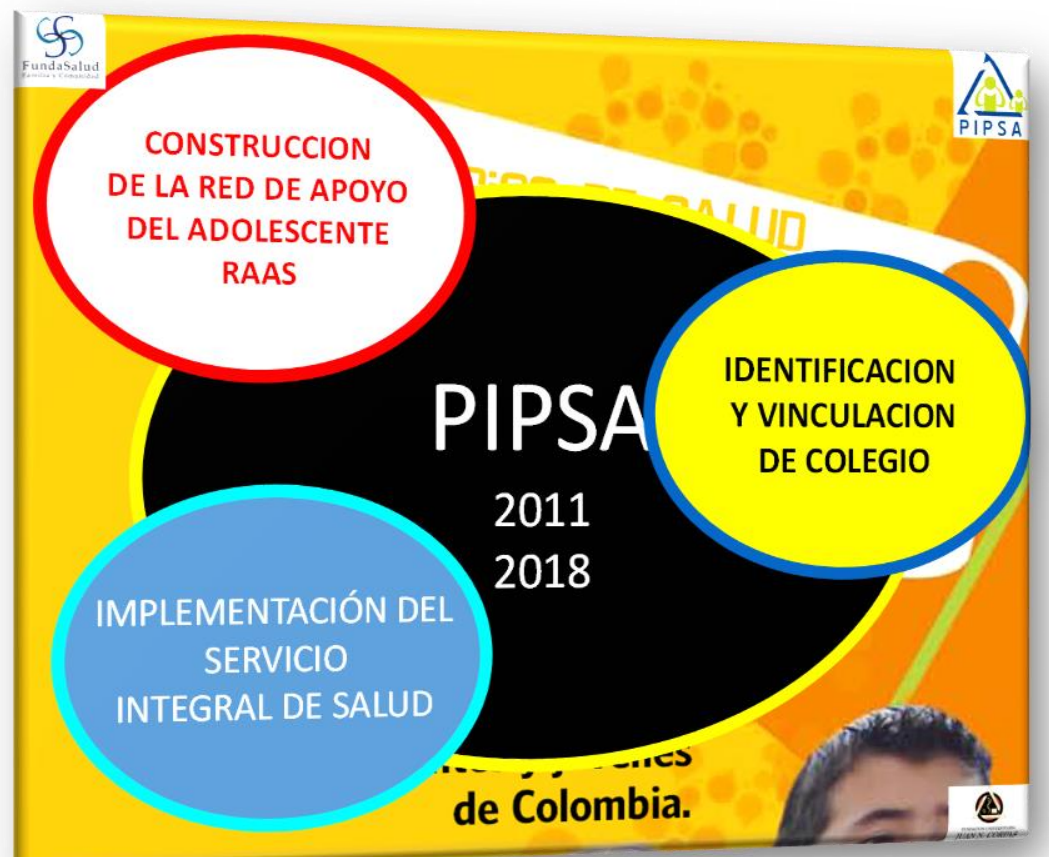

¿Cómo se puede comprometer la universidad con las comunidades donde tiene sus escenarios de práctica? Eso es precisamente el PIPSA: un programa creado en 2005 y que pretende impactar en los adolescentes a través de la intervención de la universidad.
A continuación, se presentará este programa visto a través de los ojos de uno de las docentes. Nuestra intención es que, si este artículo es leído por otras universidades, puedan, a través de lo que ha sido nuestra experiencia, vincularse con los colegios y replicarla o, a partir de la misma, organizar otras similares, pues estamos convencidos de lo mucho que se debe hacer y aprender en torno a los jóvenes.

${ }^{+\dagger}$ MD. Docente Departamento de Medicina Comunitaria FUJNC- jenny-salamanca@juanncorpas.edu.co 


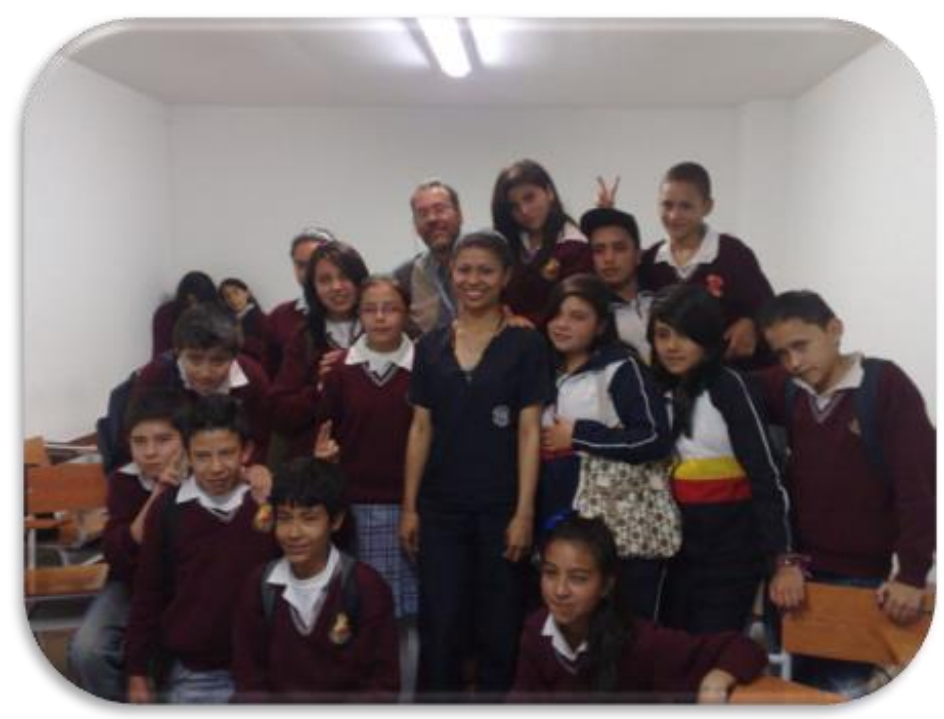

¿Cuál es la razón de este proyecto? Ni más ni menos ellos... Es comprometernos como universidad y buscar intervenciones que contribuyan al desarrollo de la población adolescente, en nuestro caso, la que está vinculada a la nuestra facultad a través de los diversos programas que venimos realizando en la localidad de Suba.

\section{Contexto y objetivos}

Fecha en la que inició el proyecto: 2005.

Tras un período de evaluación, y, además, como parte de un requerimiento académico, uno de nuestros docentes llegó a la conclusión de que, a pesar de los esfuerzos de la Fundación Universitaria Juan N. Corpas y su Departamento de Medicina Comunitaria por trabajar con la comunidad, la adolescencia, era un ciclo vital descuidado por la profesión médica. ¿La razón? Tal vez, por ser una etapa donde pocas enfermedades biológicas se presentan, poco acuden a la consulta. En cambio, existen numerosos cambios psicosociales. Dado que los médicos nos hemos preparado más para atender lo biológico, ahí se abre un distanciamiento. Este programa se crea para cerrar esa brecha y acercarnos más a la Medicina integral que tanto predicamos en nuestra facultad.

\section{Imagen de las etapas}

El primer paso consistió en hacer una extensa revisión de programas escolares por parte del personal de salud. Con base en tal revisión surgió una idea... comprometernos con un grupo de adolescentes y acompañarlos a través de su vida escolar.

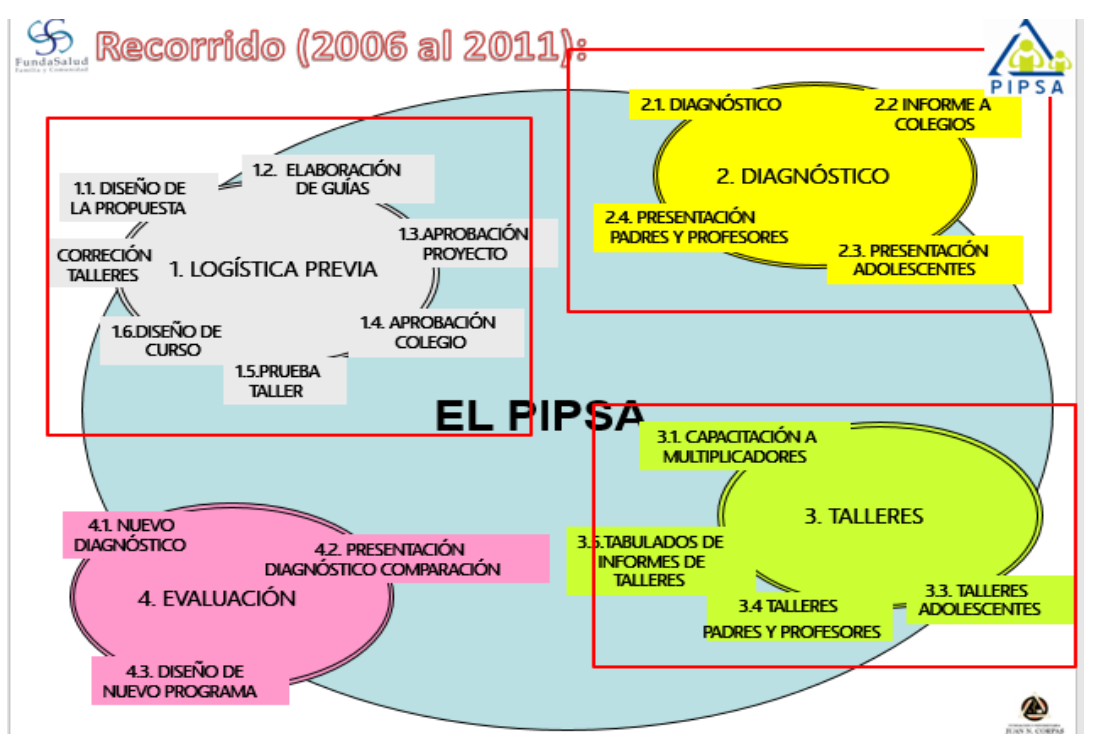

Carta Comunitaria. Vol. 26. Número 147. Abril-Junio 2018 


\section{Lo fácil: el diagnóstico}

Con el fin de reconocer el mundo de los jóvenes, se efectuó un ambicioso diagnóstico de situaciones psicosociales de los jóvenes. Se hizo con recursos y apoyo de la rectoría y con el compromiso del Departamento de Comunitaria de la FUJNC. Así surgió lo que fue la fase de diagnóstico, que servirá de línea de base en todo nuestro trabajo con los adolescentes.

\section{La acción social de relacionarnos}

Con ese estupendo insumo y sus sorprendentes resultados, comprendimos que lo que seguía era unirnos con otros sectores para propiciar cambios. Las orientadoras de los colegios, en particular algunas, se unieron con pasión, con lo que creamos las bases para construir un proyecto piloto. Ellas nos abrieron las puertas de los colegios. Entró en juego una fundación (la Fundación Salud Familia y Comunidad, financiada por la FUJNC) encargada de consolidar nuestras relaciones con la comunidad (orientadoras), la cual decidió disponer de una persona clave para ello. Cuatro docentes se vincularon, más una experta en manejo de información. Finalmente nos vinculamos con una facultad de Psicología que decidió poner a nuestro cargo dos estudiantes avanzados para realizar las intervenciones.

\section{Las intervenciones preliminares}

Inicialmente, el equipo original (donde deben estar los "dolientes", queriendo decir con ello, personas comprometidas hasta los "tuétanos"), después de buscar, leer, discutir y analizar intervenciones previas usadas en situaciones similares, aceptó el reto de realizar talleres de intervención para algunos de los jóvenes (prueba piloto), previamente diagnosticados de uno de los tantos colegios que se ofrecieron para hacer parte del proyecto. Estos talleres estaban orientados a la toma de decisiones. La metodología usada fue la de resolución de problemas hipotéticos, las sesiones se llevaron a cabo todos los sábados durante seis meses, con un grupo de adolescentes líderes. Una vez más, la Fundación Salud Familia y Comunidad junto con la rectoría de la Corpas, se comprometieron con la logística para asegurar la comodidad de los jóvenes. iEsto es clave! Atenderlos con refrigerios y ofrecerles un espacio para las discusiones, resultó fundamental. De esa primera experiencia surgió el material escrito en forma de guías para hacer las intervenciones de los jóvenes.

Un par de años después, un proyecto similar se llevó a cabo, pero en diferentes escenarios pues las intervenciones se realizaban fuera del colegio, con el fin de ofrecer a los jóvenes un cambio de ambiente que favoreciera su participación. Sin embargo, todos los involucrados en estas intervenciones sabían que estos esfuerzos no eran suficientes, un sinnúmero de limitaciones y dificultades surgieron de estos talleres; trabajar con adolescentes no era fácil. Era evidente que la preparación y la formación de equipos multidisciplinarios eran necesarios y, la continuidad era esencial para tener un impacto verdadero. En ese momento era claro que cuantas más dificultades encontrábamos, más retos debíamos y queríamos asumir.

\section{DESARROLLO}

\section{Entonces...}

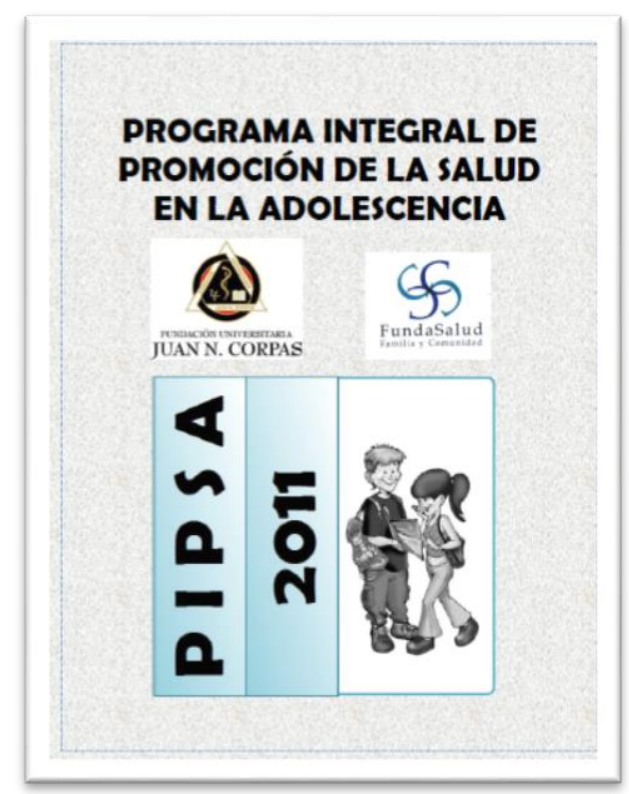

Con estos resultados, teniendo tanto una propuesta para la realización de los talleres (se crearon diferentes estrategias de intervención) como las prevalencias de los riesgos que enfrentan los adolescentes, en 2011 se logró la articulación con las(os) orientadoras(es) de los colegios de la localidad y con dos estudiantes de Psicología de la Corporación Universitaria Iberoamericana (talleristas) para programar una semana de intervenciones anuales (cuatro sesiones para cada uno de los grupos de estudiantes participantes), durante cinco años consecutivos (acompañamiento de los jóvenes durante toda la secundaria). El equipo docente del Departamento de Medicina Comunitaria se comprometió con la capacitación y el acompañamiento de los talleristas. 


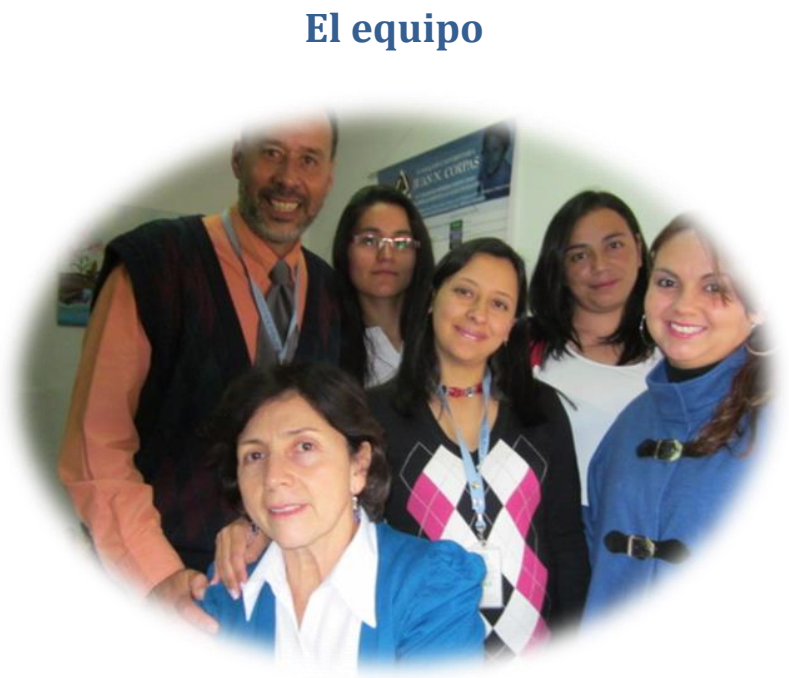

Una de nuestras docentes asumió la coordinación de las prácticas; nuestra enfermera docente, la capacitación de los estudiantes replicadores (los estudiantes de Psicología y los estudiantes de Medicina); una médica, el acompañamiento de los psicólogos; la ingeniera de sistemas, las bases de datos para el procesamiento de la información obtenida y, la comunicadora de la Fundación Salud Familia y Comunidad, el acompañamiento de las orientadoras.

\section{El "arrancón"}

Estas intervenciones iniciaron con estudiantes de 70 grado pertenecientes a tres colegios públicos de la localidad de Suba que habían hecho parte de la fase diagnóstica. Las sesiones semanales tienen como objetivo guiar al adolescente hacia la toma de decisiones asertivas al enfrentar los riesgos psicosociales encontrados en los diagnósticos previos. Los estudiantes son encuestados cada año, con el fin de conocer sus entornos y realidades, lo que nos ayuda a ajustar las intervenciones futuras $y$, a su vez, a evaluar el impacto que el programa tiene en ellos.

\section{Esos talleres iniciales y su evolución}

Se prepararon cuatro cartillas integrales para realizar la intervención en los adolescentes, combinando los mensajes y la lúdica que implicaba su ejecución. Sin embargo, a lo largo del proyecto, los estudiantes de Psicología fueron girando los talleres más a la implementación de prácticas lúdicas y videos, dado el acceso a los video beams en los colegios. De eso existe registro $y$, sin duda, es sumamente valioso pues muestra las mejores prácticas de acuerdo al momento de los adolescentes.

\section{Del diagnóstico inicial}

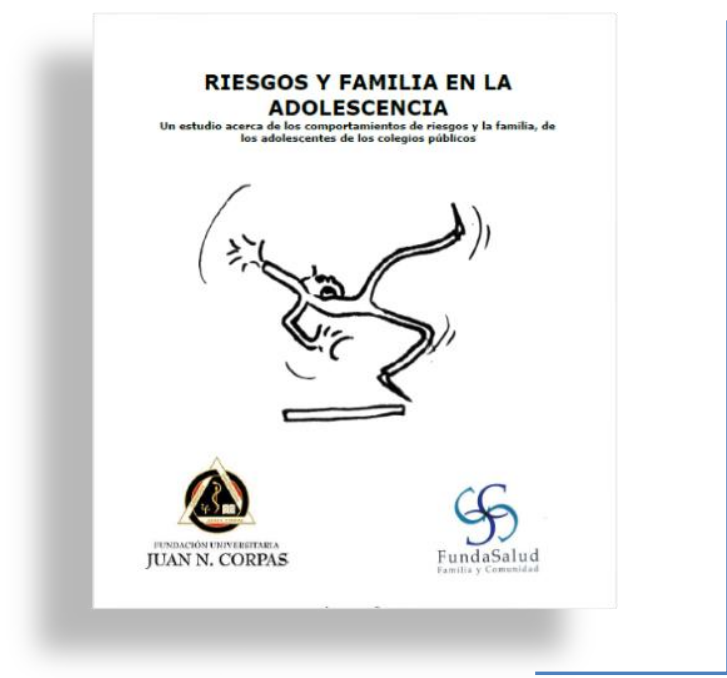

En 2005 se evaluaron diferentes cuestionarios y encuestas y, después de un par de pruebas piloto y ajustes, finalmente fueron aplicados. La información obtenida de este proceso fue abrumadora. Cerca de 13270 jóvenes, hombres y mujeres, de 12 colegios públicos de Suba respondieron los cuestionarios.

Los resultados mostraron los riesgos psicosociales a los que estos jóvenes de entre 12 y 19 años se ven enfrentados a diario. La funcionalidad familiar, la autoestima, las adicciones no farmacológicas, los conocimientos, actitudes y prácticas relacionados con el uso de sustancias psicoactivas, la violencia, los desórdenes de alimentación, la salud sexual y reproductiva y, la depresión y el pensamiento suicida, fueron las temáticas evaluadas en este diagnóstico. 


\section{Papel de la familia}

Estos primeros resultados mostraron que la funcionalidad familiar puede ser un factor protector o de riesgo; $66 \%$ de los jóvenes encuestados no conocían o no entendían el ciclo menstrual o su relevancia, teniendo un conocimiento mínimo y casi nulo acerca de los métodos de planificación familiar. $32 \%$ de los adolescentes estaban en alto riesgo de padecer desórdenes de alimentación.
Uno de los resultados más impactantes fue que, el $40 \%$ de ellos ya habían incurrido en actos violentos. Además, cerca del $17 \%$ ya habían experimentado problemas relacionados con el uso del cigarrillo y/o del alcohol.

Este tipo de diagnósticos se repitió en 2006 y, de nuevo en 2011, con el fin de evaluar la realidad adolescente y sus cambios a través del tiempo. Luego de estos hallazgos, el siguiente paso era evidente. iEra el momento de intervenir!

\section{Lo cierto}

iEstamos abrumados de información! y se ha creado la necesidad de abrir espacios en nuestro currículo para analizar todo lo que hemos conseguido. Hemos procurado presentar informes a los colegios, a los padres, a la localidad, pero somos conscientes de que, a la fecha, solo hemos utilizado una mínima parte de todo lo encontrado.

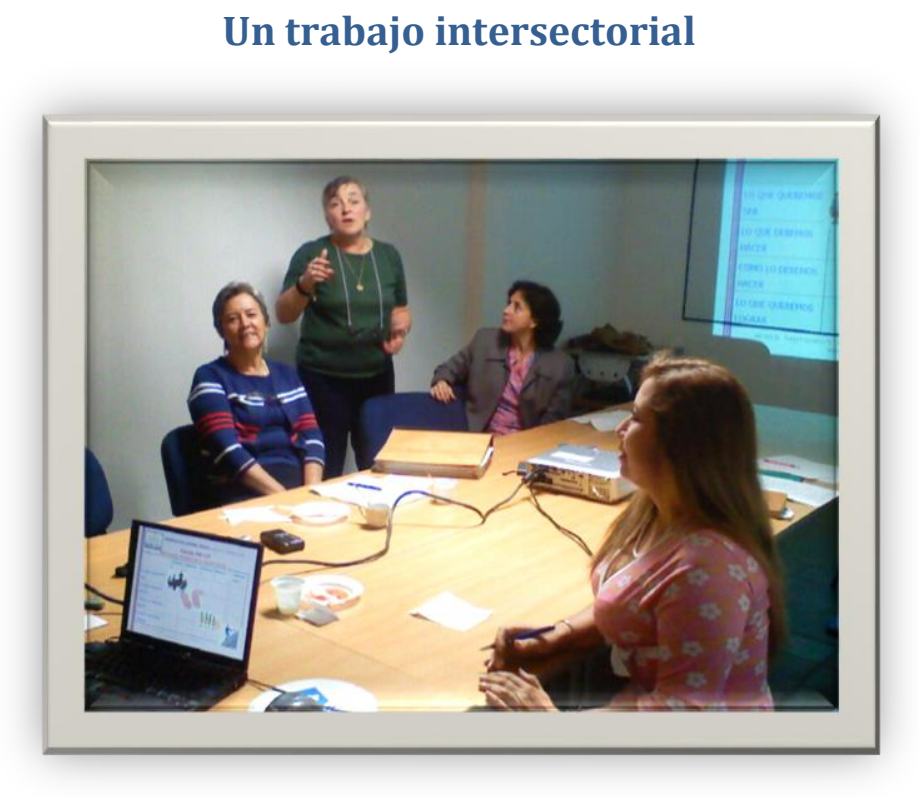

Vale la pena contar que esta experiencia, el PIPSA, en sus primeros cinco años buscó la ayuda de diferentes entidades públicas y privadas en pro de la adolescencia. En tal sentido, en el año 2007 se creó el RAAS (Red de Apoyo al Adolescente de Suba) y así empezamos a unir fuerzas con el fin de ayudarnos los unos a los otros en las dificultades que se presentaban al abordar problemáticas adolescentes. Hemos intentado el mantenimiento del RAAS (se trata de personas vinculadas a entidades diferentes y que tienen como objetivo común apoyar a los adolescentes).

Pero, icarambas! ha sido difícil sostener y darle continuidad a esta red. A las personas de estas entidades las reasignan a otros lugares y eso afecta el compromiso hacia esta red. Aun así, algunas de sus reuniones mensuales (hemos sido constantes) nos ayudan al unir fuerzas para dar soluciones a diferentes problemáticas y dificultades a las que se ven enfrentados nuestros adolescentes y sus familias.

Ciertamente hemos podido mantener las redes de apoyo con diferentes instituciones y asegurar la continuidad del programa. Hemos tenido la fortuna de trabajar con entidades como el Hospital de Suba, la Red Unidos, el CADEL, la Fundación Gamma Idear, la Corporación Universitaria Iberoamericana, las universidades Santo Tomás y Manuela Beltrán, la Fundación Salud, Familia y Comunidad y, por supuesto, nosotros en representación de la Fundación Universitaria Juan N. Corpas y su Departamento de Medicina Comunitaria. Tener una persona a cargo de esto resulta fundamental.

\section{Sin embargo...}

Reconocemos que la consolidación de estas personas y de las entidades que representan está siendo sometida a una desbandada dado que el compromiso, y lo repetimos, ha sido más personal que de las instituciones y cuando la persona cambia de sitio, encontrar otra que se "comprometa" no ha sido la pauta. No por ahora, pero lo seguiremos intentando... iEste es uno de los retos! 
Desde que pudimos formar un equipo multidisciplinario con los estudiantes de Psicología, la fase de intervención se convirtió en realidad en 2011. Teníamos experiencia previa y conocíamos las problemáticas que se debían abordar gracias a los resultados de la fase diagnóstica y, después de analizar y discutir, decidimos continuar con la metodología de toma de decisiones asertivas centradas en cinco temáticas básicas:

1. Violencia y resolución de conflictos.

2. Pubertad, salud sexual y reproductiva.
3. Consumo y abuso de sustancias psicoactivas y adicciones no farmacológicas.

4. Autoestima y depresión.

5. Trastornos de alimentación.

Nosotros, como equipo médico, proveemos a los estudiantes de Psicología las bases biológicas y médicas de las problemáticas mencionadas; ellos en retorno nos ayudan con los aspectos psicológicos y emocionales, así como con el manejo didáctico de las sesiones.

\section{El "arrancón"}

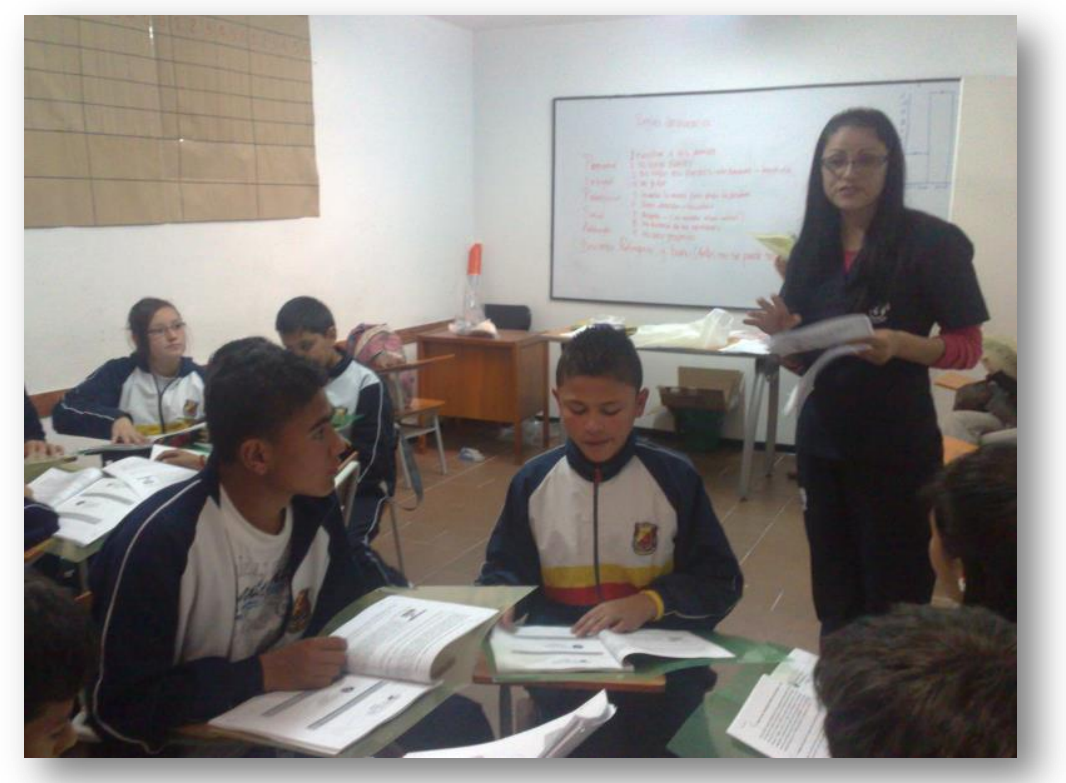

Así, dimos inicio a la intervención. Comenzamos con tres colegios, lo que se tradujo en cerca de 360 adolescentes que cursaban séptimo grado. Los talleres consistían en cuatro sesiones para cada grupo (curso) en cada colegio. Los estudiantes se dividieron en pequeños grupos de 4 o 5 personas, para trabajar en competencia entre ellos en diferentes actividades lúdicas, bien fuera dibujar, discutir, actuar o escribir acerca de la temática propuesta.

Al finalizar cada sesión, se aplicaron cuestionarios similares a los usados en la fase diagnóstica. En ese primer año (2011) el promedio de edad de los adolescentes fue de 12,9 años. Los grupos estaban conformados por un $48 \%$ de hombres y $51 \%$ de mujeres. Algunos de los hallazgos durante esta evaluación fueron:
1. El $30 \%$ había consumido alcohol en la última semana.

2. EL $5 \%$ había tenido contacto previo con marihuana y el $1 \%$ con cocaína.

3. El $78 \%$ tenía autoestima alta.

4. El $12 \%$ tenía acceso a armas de diferentes tipos.

5. El $14 \%$ estaba en riesgo de depresión y el $10 \%$ de trastornos de alimentación.

6. Un mínimo conocimiento acerca de los cambios físicos y funcionales de la pubertad, así como del ciclo menstrual.

\section{La continuidad}

Ahí está uno de nuestros éxitos. Gracias a los mismos estudiantes de Psicología que abrieron el PIPSA en sus universidades como un campo de práctica, cada año el grupo de psicólogos entrega a otros el proceso y, eso, junto con nuestro acompañamiento, permite la continuidad del proceso. Las reuniones de nuestro equipo con los tutores de Psicología de los estudiantes son claves. Todo esto le da la seriedad suficiente al proyecto como para sostenerse académicamente. 


\section{Con los adolecentes}

Una de las ventajas ha sido, hasta el momento, el que los jóvenes recuerdan fácilmente lo trabajado en el año anterior y tenemos pruebas de que les gusta (sumado al hecho de que les encanta no hacer las clases habituales que el PIPSA reemplaza). En el polo opuesto, una de las dificultades encontradas, estaba relacionada con la alta rotación de estudiantes, pues no siempre seguían en el mismo colegio o habían cambiado de grupo por pérdida de sus deberes académicos.

Un reto es diseñar la metodología pertinente para cada año y tener el cuidado de abordar las temáticas de forma diferente, pero, con el mismo objetivo preventivo. Aquí reconocemos que son los estudiantes de Psicología los que llevan la batuta. Ellos a través de ese contacto con los jóvenes van generando las metodologías.

Lo cierto es que, para nuestra sorpresa, la última medida de 2015 nos mostró que las prevalencias de riesgo de los adolescentes que esperábamos modificar no alcanzaron las expectativas que teníamos. Nos reconforta saber que la participación es bien respaldada por los mismos adolescentes y que los padres y las directivas del colegio, a través de las orientadoras se muestran más comprometidos. Pero nos falta analizar más lo conseguido $\mathrm{y}$, tal vez, reforzar aspectos claves.

\section{¿Qué ha resultado de este proyecto?}

Desde la creación del PIPSA, hemos podido monitorear la información básica obtenida en la fase inicial de diagnóstico y, aunque no hemos podido medir (por ahora) la disminución o aumento de los riesgos a los que se ven enfrentados nuestros adolescentes, sí hemos podido ver (percepción) el cambio en su proceso de toma de decisiones. En el comienzo, muchos de ellos no sabían a dónde ir o a quién buscar por ayuda o asesoría al enfrentarse a este tipo de riesgos psicosociales, al igual que por situaciones que generaran en ellos vergüenza o pena, lo que los llevaba a guardarse muchas de sus dudas y preguntas. Ahora, después de casi 10 años de trabajar con adolescentes, podemos ver que la comunicación entre ellos, las orientadoras(es), sus padres y el equipo médico y psicológico fluye en diferentes direcciones, colaborando en soluciones integrales para cada caso, lo que para nosotros es uno de los más grandes logros del programa. Creemos que construimos un laboratorio social por demás interesante.

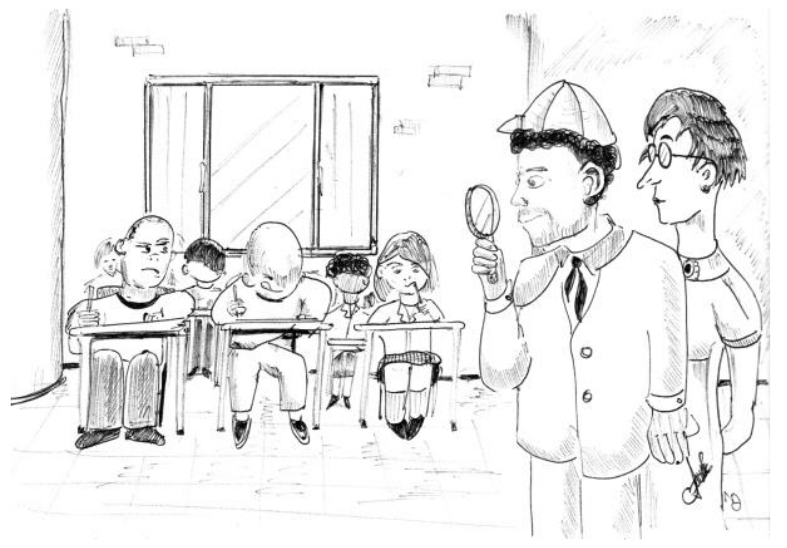

\section{¿Cómo medir?}

Estamos en esa tarea. Queremos creer que nuestro programa ha influenciado la vida de estos jóvenes. Pero ¿qué tanto? No lo sabemos. Propusimos medir los cambios en las encuestas perceptivas, pero, al revisarlas, no encontramos las medidas que esperábamos. Estamos tratando de interpretar los cambios conseguidos y sabemos que aun debemos analizar más y más. Lo que, sin duda, hemos aprendido, son distintas metodologías para el abordaje de adolescentes. Nuestra decisión ha sido, y sigue siendo, continuar, pues creemos que, sobre la marcha, iremos ajustando.

\section{Un logro: la retroalimentación comunitaria}

\section{EN TORNO A LA ESTRUCTURA FAMILIAR}

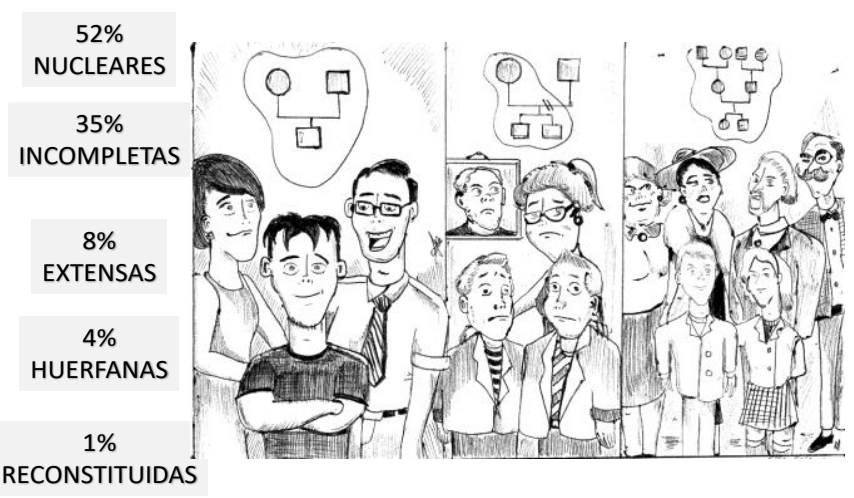

Cada intervención en alguno de los cursos de los colegios participantes, genera un informe de las encuestas aplicadas. Este se entrega a las orientadoras; además, se ha implementado una presentación a los padres de los jóvenes, encontrando realmente muy interesante la sorpresa que se llevan en particular con los más pequeños, al identificar situaciones que no se esperaban (por ejemplo, inicio de vida sexual).

Tales presentaciones han resultado claves para que los padres se comprometan, al menos con la autorización del programa del siguiente año. Desde ya tenemos en mente la necesidad de hacer una escuela de padres, que se prepare con base en los resultados para seguir acompañando a sus hijos. Sabemos que, si la logramos construir y además unimos a los profesores, la fuerza del programa se incrementa y con ella, la posibilidad de ofrecerles más oportunidades a estos adolescentes. 


\section{¿Cuál es el estado actual del proyecto? ¿Y el futuro?}

Ya terminamos (diciembre de 2015) las intervenciones de esta primera cohorte de adolescentes. Tenemos toda la información. Hemos empezado los análisis de resultados y estamos revisando. Por asuntos de cambios estructurales en nuestro departamento, nos hemos tomado más tiempo del que creíamos para el análisis y por ello, y para no perder el compromiso que tenemos con los colegios, hemos iniciado una nueva cohorte (febrero de 2016) y ya estamos en el segundo año.

Estamos sorprendidos de la inmensa cantidad de información recogida y sabemos que tenemos el reto de presentarla y con base en ella hacer las adecuaciones pertinentes.

\section{Lo que hemos reiniciado}

Las cinco temáticas básicas no han cambiado y a través de los años hemos tenido la oportunidad de identificar y poner en práctica diferentes estrategias para abordarlas.

Hemos aprendido que intervenciones como esta, en la que los adolescentes reciben un abordaje integral de sus riesgos y realidades, pueden tener un mayor impacto (intuición) que los procesos convencionales de conferencias donde el profesor explica y el joven oye.

Somos conscientes de que nos ha faltado el cierre formal según lo propuesto en la cohorte iniciada en 2011, pero, celebramos que, a pesar de ello, iniciamos una nueva cohorte para no perder nuestro compromiso hacia los adolescentes.

Sabemos que tenemos ante nosotros la oportunidad de cumplir uno de los objetivos de este programa, como es el de tener la habilidad de formar replicadores en los estudiantes de Psicología, en los maestros y orientadores(as) que nos han ayudado a través de los años $y$, por supuesto, en los padres y en los mismos adolescentes buscando así la expansión del programa que el equipo de inicio esperaba cuando comenzó la fase diagnóstica.

\section{Un reconocimiento}

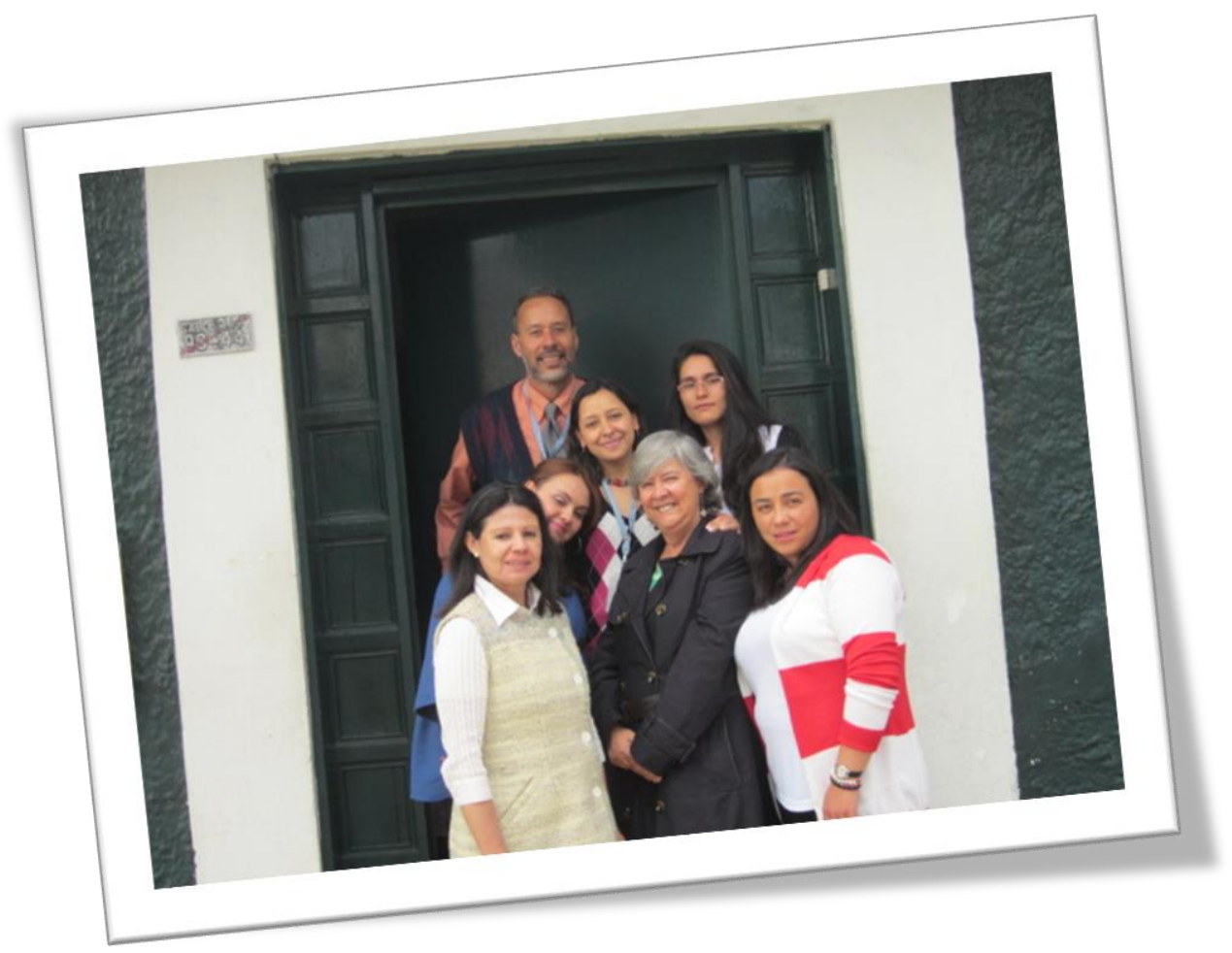

Las redes de apoyo creadas a través de este programa con las facultades de Psicología, los colegios, las orientadoras(es) y maestros y nosotros en pro del bienestar de los adolescentes se han mantenido durante los cerca de 10 años de actividad que lleva el programa y, esperamos, que continúen así. Como quien dice, acá estamos y no nos vamos. 


\section{CONCLUSIONES}

\section{¿Por qué funciona el PIPSA?}

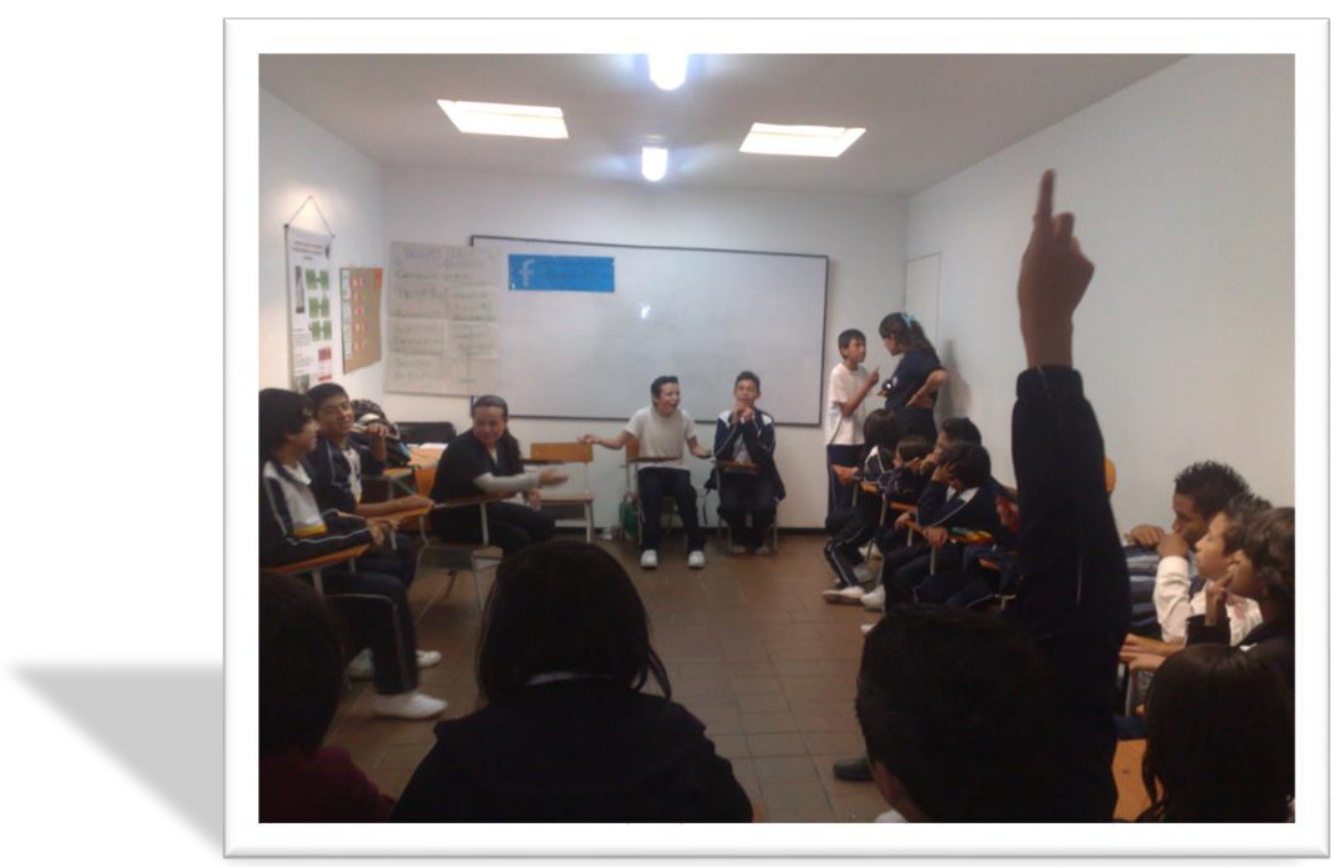

Este proyecto funciona, primero, gracias a las orientadoras y orientadores de los colegios públicos y a su compromiso con sus estudiantes; han trabajado arduamente a nuestro lado durante los últimos diez años hasta el día de hoy.

Segundo, gracias a los convenios que hemos podido realizar con las facultades de psicología de la Universidad Manuela Beltrán (y ahora estamos tras uno con la Universidad Minuto de Dios), quienes, cada semestre, nos han ayudado a dar continuidad al programa.

En tercera instancia, el PIPSA funciona gracias a la dinámica lúdica usada en los talleres por los psicólogos (figuras estelares de este proyecto), que permiten que los conceptos se formen de manera constructivista, para que los adolescentes los recuerden y pongan en práctica cada año, a pesar de sus dudas en ciertas áreas.
Finalmente, a través de los años, hemos logrado una mejor conexión con los padres de familia, quienes al comienzo no asistían a las charlas, talleres o actividades preparadas para ellos; de aproximadamente 120 padres, solo asistían 5 o 10 máximo; ahora podemos trabajar con un $50 \%$ a $60 \%$ del total de padres invitados.

Este programa puede funcionar en otros contextos; puede ser replicado en diferentes instituciones educativas o incluso en diferentes departamentos o países, por la sencillez de su metodología que permite a cada adolescente un aprendizaje significativo, necesitando únicamente el compromiso de otras facultades.

Para 2018, tenemos el reto de consolidar lo conseguido, producir los informes pertinentes $y$, por, sobre todo, promover que otras universidades se vinculen a proyectos similares. Nosotros estaremos listos a compartir todo lo aprendido.

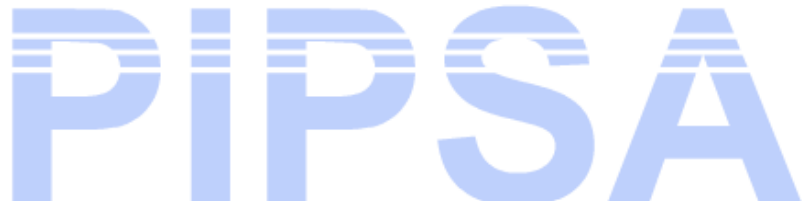

PROGRAMA INTEGRAL DE PROMOCION DE SALUD DEL ADOLESCENTE 